\title{
Editorial
}

\section{Feuer unter dem Herd der klinischen Ernährungstherapie}

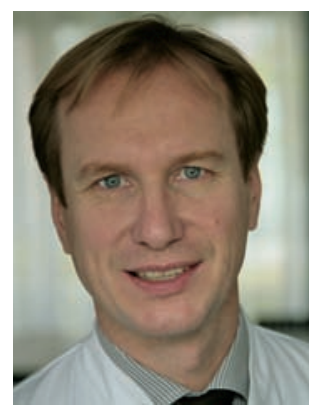

1974 beschrieb Charles E. Butterworth in seinem Artikel „The Skeleton in the Hospital Closet" an Hand von fünf Krankengeschichten die negativen Auswirkungen einer unzureichenden Ernährungstherapie auf die Krankheitsdauer und das Überleben der Patienten (Nutrition Today 1974; 4-8). Diese Publikation war der Funke, der das Feuer in der Küche der Ernährungsmedizin entfachte. Die öffentliche und wissenschaftliche Diskussion über die inakzeptabel große Zahl mangelernährter Klinikpatienten in Europa und den gravierenden Folgen von Mangelernährung - verlängerte Klinikaufenthalte, verzögerte Rehabilitation, verringerte Lebensqualität, erhöhte Kosten - kulminierte in der Resolution des Europarates vom Dezember 2003 über die Verpflegung und Ernährungsversorgung in Krankenhäusern. In dieser Resolution werden bereits die wichtigsten Eckpunkte zur Etablierung einer systematischen Ernährungstherapie aufgezeigt:

- Routinemäßige Erfassung des Mangelernährungsrisikos mit einfachen Methoden

- Erkennung und Vermeidung möglicher Ursachen von Mangelernährung

- systematische Ernährungstherapie als Teil jeder Behandlung

- Priorität für die Ernährung mit normalen Lebensmitteln

- künstliche Ernährung, wenn die normale Ernährung nicht ausreichend ist.

So banal dies klingen mag, scheiterte aber die Umsetzung dieser Richtlinie in der Praxis häufig bereits am ersten Punkt der Liste, da es meist nicht gelang, die Erfassung der Mangelernährung in die Aufnahmeroutine zu implementieren. Die ärztliche Arbeit ist in der Regel an kategorisierbare Parameter als Entscheidungsgrundlagen gekoppelt, so führt z.B. erst ein erhöht gemessener Blutdruck zu bestimmten Untersuchungen und zur Einleitung einer antihypertensiven Therapie. In gleicher Weise wird der Arzt (als Teil des Ernährungsteams) erst auf konkrete Ergebnisse aus Screeningtools wie dem NRS (Nutritional Risk Screening 2002) oder SGA (Subjective Global Assessment) mit diagnostischen und therapeutischen Entscheidungen reagieren.

In diesem Zusammenhang ist der in dieser Ausgabe der E\&M beschriebene Expertenstandard zum Thema „Ernährungsmanagement zur Sicherstellung und Förderung der oralen Ernährung in der Pflege“ als Meilenstein in der klinischen Ernährungstherapie anzusehen, da er den Screeningprozess als wichtigen Teil der Aufnahmeroutine etablieren hilft (S. 116). Mit dem kategorisierten Ergebnis dieses Aufnahmescreenings (A: kein, B: erhöhtes oder C: hohes Ernährungsrisiko) hat der behandelnde Arzt einen Handlungsauftrag. Dieser Handlungsauftrag muss als ersten Schritt eine Ursachenforschung bewirken. Dieses weite Spektrum der Ursachen einer Mangelernährung haben Koch et al. in ihrer Übersicht auf Seite 111 dargestellt. Als zweiten Schritt muss der Arzt prüfen, ob und welche Ernährungstherapie notwendig ist. Ein dritter wichtiger Schritt fehlt in den Eckpunkten der Resolution des Europarates: Die Kontrolle, ob die verordneten ernährungstherapeutischen Maßnahmen „im“ Patienten angekommen sind und sich damit der Ernährungszustand auch gebessert hat. An diesem Punkt muss in Zukunft noch nachgearbeitet werden. Auf dem Feuer aber, das Dr. Butterworth entfacht hat, wird mittlerweile eine immer bessere Kost gekocht, die hoffentlich bald alle satt macht.

Priv.-Doz. Dr. Edmund A. Purucker 